\title{
Tau in MAPK activation
}

\section{Chad J. Leugers, Ju Yong Koh, Willis Hong and Gloria Lee*}

Department of Internal Medicine, University of lowa Carver College of Medicine, lowa City, IA, USA

\section{Edited by:}

Naruhiko Sahara, National Institute of Radiological Sciences, Japan

Reviewed by:

Jurgen Gotz, The University of Sydney, Australia

Irving E. Vega, University of Puerto

Rico-Rio Piedras Campus, Puerto Rico

*Correspondence:

Gloria Lee, Department of Internal

Medicine, University of lowa Carver

College of Medicine, 500 Newton

Road, ML B191, lowa City, IA 52242, USA

e-mail: gloria-lee@uiowa.edu

The nature of "toxic" tau in Alzheimer's disease (AD) has been unclear. During pathogenesis, the importance of tau oligomerization vs. tau phosphorylation is controversial and the investigation of both remains critical toward defining the "toxicity" of tau. The phosphorylation of tau on serines and/or threonines occurs early in the disease course and altering phosphorylation has been shown to disrupt neuropathogenesis. We have recently reported that in PC12-derived cells, tau had a role in signal transduction processes activated by NGF. By depleting tau, NGF-induced MAPK activation was attenuated and by restoring tau, MAPK activation was restored. Furthermore, the phosphorylation of tau on Thr231 was required for tau to potentiate MAPK activation. Here we report the effects of additional disease-related tau phosphorylation sites and tau isoform on the ability of tau to potentiate MAPK activation. Our findings, which tested three other sites of phosphorylation, showed that phosphorylation at these other sites mainly lessened MAPK activation; none potentiated MAPK activation. In comparing ON3R tau to the other five brain tau isoforms, most showed a trend toward less MAPK activation, with only 2N4R tau showing significantly less activation. Since MAPK activation has been reported in AD brain and is characteristic of cell proliferation mechanisms, tau phosphorylation that promotes MAPK activation could promote cell cycle activation mechanisms. In neurons, the activation of the cell cycle leads to cell death, suggesting that abnormally phosphorylated tau can be a toxic species. The relationship between tau oligomerization and its ability to potentiate MAPK activation needs to be determined.

Keywords: tau, MAPK activation, phosphorylation, signal transduction, NGF

\section{INTRODUCTION}

The existence of tau pathology occurs in many age-related neurodegenerative diseases that are now termed "tauopathies." Among these diseases, Alzheimer's disease (AD) is the most prevalent and it has been suggested that the presence of tau is critical for disease progression $(1,2)$. Neurodegenerative diseases caused by both missense and intronic mutations in the tau gene have indicated the ability of tau to cause disease [reviewed by Ref. (3-5)]. However, the mechanism by which tau leads to neurodegeneration is unknown. For instance, whether there is a loss of function or a gain of toxic function remains controversial. In considering the role of different tau species during neurodegeneration, hyperphosphorylated tau, and tau filaments have long been investigated. Evidence suggesting that neurofibrillary tangles were not a toxic species came from data indicating that tau-induced behavioral deficits could be improved without changing the tangle burden (6). In fact, neuronal loss did not correlate with neurofibrillary pathology (7). Also, in Drosophila and C. elegans, tau-induced neurodegeneration occurred in the absence of neurofibrillary tangles $(8,9)$. Most recently, the investigation of tau oligomers has suggested that they may have an early role in neurodegeneration. Tau oligomers correlate with cellular abnormalities (10-12) and neurodegenerative disease (13-16). However, the molecular mechanism by which tau oligomers cause toxicity has not been clearly demonstrated. In addition, in these studies, the tau oligomers were composed of phosphorylated tau, making it difficult to isolate the effects of oligomerization from those of phosphorylation.
Tau phosphorylation is required for its neurotoxic effects (17, 18) and as tau is hyperphosphorylated early in the disease process, it is not surprising that tau oligomers would be formed from phosphorylated tau. Therefore, in determining if tau oligomers have specific function, one could also first determine the function of abnormally phosphorylated tau, then ask if that tau was in the form of oligomers. Recently, we found that tau has the ability to potentiate NGF-induced MAPK activation and that phosphorylation on Thr231 was critical for the activity (19). Since this activity was seen within $3 \mathrm{~h}$ after NGF addition, our data identified a new role for tau in signal transduction processes that take place during neuronal differentiation. At the same time, as Thr231 is phosphorylated early during neurodegeneration (20), it raised the question of whether this new tau activity had a role in neurodegeneration. To further probe the relationship between tau phosphorylation and its ability to potentiate MAPK activation, here we investigate the effects of additional phosphorylated sites, focusing on sites relevant to AD. We also investigate the effects of alternative splicing on the ability of tau to affect MAPK activation.

\section{MATERIALS AND METHODS CELL CULTURE}

PC6-3 cells (21) were cultured on collagen (BD Biosciences) coated dishes using RMPI 1640 medium with 10\% horse serum and 5\% fetal bovine serum. D5 cells, a stable PC6-3 cell line with stable over-expression of the $0 \mathrm{~N} 3 \mathrm{R}$ isoform of human tau, and rTau4 cells, a PC6-3 cell line with stable expression of hairpin RNAi 
targeting endogenous rat tau, were previously described (19). Media for stable cell lines was supplemented with $200 \mu \mathrm{g} / \mathrm{ml} \mathrm{G418.}$

\section{MAPK REPORTER ASSAYS}

MAPK activation was measured by a luciferase reporter assay as described by Leugers and Lee (19). The ability of tau mutants to influence NGF-induced MAPK activation was studied by cotransfecting tau plasmids with the MAPK reporter plasmids. Tau plasmids used were pRc/CMV-0N3R, pRc/CMV-0N3R-S214D, pRc/CMV-0N3R-S404D, pRc/CMV-0N3R-S396D/S404D, pRc/ CMV-0N3R-S202D, pRc/CMV-0N3R-S199D/S202D, pRc/CMV0N4R, pRc/CMV-0N4R-S202D, pRc/CMV-0N4R-S199D/S202D, pRc/CMV-1N3R, pRc/CMV-2N3R, pRc/CMV-1N4R, and pRc/ CMV-2N4R. (0N3R, 0N4R, etc., denote tau isoforms where 0N3R contains 352 residues with no amino terminal inserts and three microtubule binding repeats; $2 \mathrm{~N} 4 \mathrm{R}$ contains 441 residues with two amino terminal inserts and four microtubule binding repeats, etc.). Mutant tau plasmids with phospho-mimicking $S$ to D mutations were constructed using site-directed mutagenesis (Stratagene, Inc.); sequences were confirmed by DNA sequencing.

\section{TAU DETECTION IN CELL LINES}

D5 cells were grown with or without NGF for 30 min and then harvested in RIPA buffer with protease and phosphatase inhibitors (19). After rocking at $4^{\circ}$ for $20 \mathrm{~min}$, lysates were centrifuged $20 \mathrm{~min}$. Supernatants were added to an equal volume of $2 \times$ Laemmli sample buffer and boiled $5 \mathrm{~min}$. Cell lysate samples were subject to SDS-PAGE and transferred to PVDF membranes. Membranes were probed with anti-phospho-Ser214-tau (Invitrogen, Inc.), PHF1 (22), AT8 (23), Tau5 (24), Tau12 (25), or anti-GAPDH (Chemicon, Inc.). Signal was visualized using ECL (Western Lightning Plus-ECL, Perkin Elmer, Inc.).

\section{RESULTS AND DISCUSSION}

To examine MAPK activation, PC6-3 cells, a PC12-derived cell line (21), were treated with NGF. To probe tau function, we used the rTau4 cell line, a PC6-3-derived cell line that expressed a hairpin shRNA that selectively down-regulated the expression of endogenous rat tau without affecting the expression of human tau mediated by transfection (19). In tau-depleted rTau4, NGF-induced MAPK activation was attenuated and the addition of wild-type human tau (0N3R) was able to significantly restore MAPK activity after growth factor treatment (19). Moreover, a phospho-mimicking mutation at Thr231 brought further increases to MAPK activation while a Thr to ala mutation at Thr231 showed a dominant negative effect on MAPK activation (19). This led us to conclude that tau phosphorylation at Thr231 was required for the effect of tau on MAPK signaling. Based on these findings, and the fact that tau can undergo phosphorylation at a number of sites during early brain development (26) or during neurodegeneration (27), we further investigated the effects of tau phosphorylation on MAPK activation.

To select tau phosphorylation sites to test, we sought sites that were modified in both AD and in the PC6-3 cells. We chose to examine Ser214, Ser396/Ser404, and Ser199/Ser202, all of which are known to be phosphorylated in $\mathrm{AD}$. While tau phosphorylation in NGF-treated PC12 cells has been examined, NGF treatments greater than $24 \mathrm{~h}$ were often used and we were interested in earlier time points as our focus was on signal transduction rather than neurite outgrowth. To assess the phosphorylation of both endogenous rat tau and exogenously expressed human tau (0N3R), we utilized the previously described PC6-3-derived cell line D5, that stably expresses human tau (19). In both undifferentiated cells and cells stimulated with NGF, we observed phosphorylation at Ser199/Ser202, detected by AT8, as well as phosphorylation at Ser396/Ser404, detected by PHF1 (Figure 1). NGF treatment was performed for $30 \mathrm{~min}$ and no further changes in the level of phosphorylation at these sites were observed for up to $3 \mathrm{~h}$ (data not shown). The phosphorylation at Ser396/Ser404 was found to occur in both rat tau and human tau species and the addition of growth factor appeared to slightly increase the level of phosphorylation. Phosphorylation at Ser199/Ser202 was also observed in both rat and human tau and did not appear to change upon NGF addition. In contrast, phosphorylation at Ser214 appeared increased after NGF induction (Figure 1). Our previous data had shown that phosphorylation at Thr231 also increased after NGF induction (19). These data indicate that these tau sites are being phosphorylated in PC6-3 cells.

To examine MAPK activation, we tested a panel of phosphomimetic mutations at these tau phosphorylation sites. In advance of measuring MAPK activation, protein produced by each plasmid was visualized by western blotting in order to confirm that equivalent amounts of each protein was being expressed in each experiment (Figure 2A). In this way, differences in MAPK activation would be attributed to protein identity rather than protein quantity. In each experiment, WT tau was expressed in the tau-depleted rTau 4 cells as control. When expressing 0 N3R human tau with a phospho-mimicking mutation at Ser214 (S214D, Figure 2B), we observed a significant attenuation of MAPK activation relative to WT tau. Next, we tested phosphorylation at Ser404 using S404D and observed a trend of reduction in MAPK signaling (Figure 2C), indicating that phosphorylation at this site might also impair the ability of tau to enhance MAPK signaling. As phosphorylation at Ser404 often occurred in conjunction with phosphorylation at Ser396 $(22,26,27)$, we also tested a double

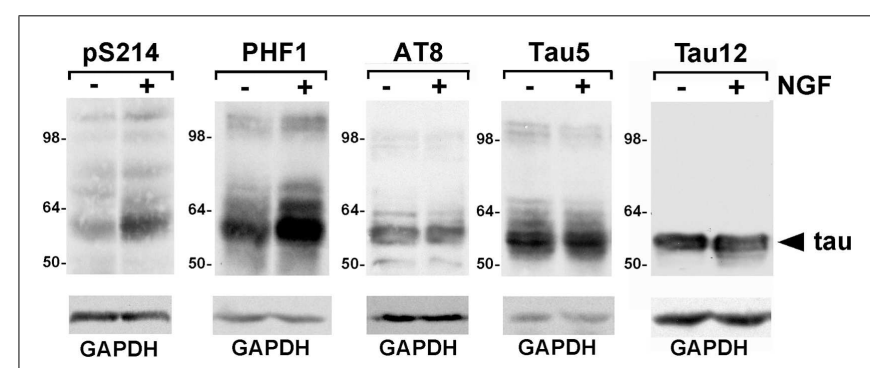

FIGURE 1 | Phosphorylated tau is expressed in PC6-3 cells. Serum starved D5 cells were stimulated with $50 \mathrm{ng} / \mathrm{ml} \mathrm{NGF}$ for $30 \mathrm{~min}$. Cells were harvested as described in Section "Materials and Methods." Following SDS-PAGE, immunoblotting was performed with antibodies as indicated, total tau being probed by Tau5 and human tau by Tau12.

Glyceraldehyde-3-phosphate dehydrogenase (GAPDH) levels are shown as a loading control. Arrowhead indicates human tau; the less abundant rat tau was visualized with Tau5. 


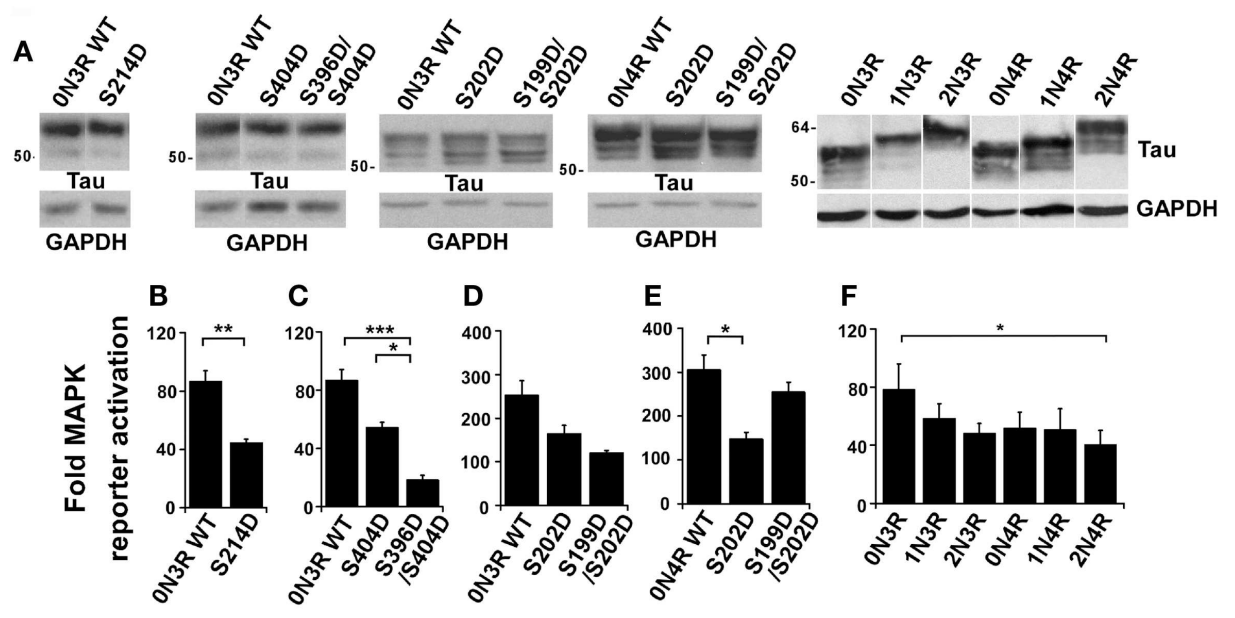

FIGURE 2 |Tau phosphorylation modulates the effect of tau on NGF-induced MAPK signaling. rTau4 was transfected with MAPK reporter system plasmids and tau plasmid indicated. Thirty-six hours after transfection, NGF was added $3 \mathrm{~h}$ prior to cell harvest. (A) To assure equal tau expression in transfections to be assayed in (B-F), lysates from rTau4 cells transfected under identical conditions were probed with Tau13.
Glyceraldehyde-3-phosphate dehydrogenase (GAPDH) levels are shown as a loading control. (B-F) Fold-MAPK reporter increase was calculated as previously described (19). ( $\left.{ }^{*} p<0.05 ;{ }^{* *} p<0.005 ;{ }^{* *} p<0.001\right)$. Data shown are mean + SE from three independent experiments; for each experiment, transfections were performed in triplicate for each condition. mutant, S396D/S404D, and found MAPK signaling significantly impaired relative to both control WT tau and S404D mutant (Figure 2C). In addition, the phospho-mimicking substitution at Ser202 was tested and we observed a trend of decreasing MAPK activation (Figure 2D). The double mutant S199D/S202D was also tested and we found a similar trend (Figure 2D). Together, these findings identified several tau phosphorylation sites where phosphorylation appeared to decrease the ability of $0 \mathrm{~N} 3 \mathrm{R}$ tau to potentiate MAPK activation. Moreover, these findings indicated that the ability of phospho-Thr231-tau to increase MAPK activation was unique (Table $\mathbf{1}$ ).

Tau mutations that affect the alternative splicing of tau mRNA can result in increased levels of $4 \mathrm{R}$ tau and cause neurodegenerative disease [reviewed in Ref. $(28,29)$ ]. To determine if the isoform identity could alter the ability of tau to affect MAPK signaling, we tested different isoforms of tau for their ability to rescue MAPK activation in rTau4. In comparing the abilities of the wild-type ON3R and 0 N4R tau to restore MAPK signaling, while significant differences were not demonstrated, there was a trend showing that $0 N 4 R$ tau had reduced activity (Figure 2F). In addition, in both $3 \mathrm{R}$ and $4 \mathrm{R}$ isoforms, we observed a trend of decreased MAPK activation as the $\mathrm{N}$-terminal inserts were added (Figure 2F). A significant difference between the largest and smallest isoforms of tau (2N4R vs. 0N3R tau) was observed (Figure 2F). These observations demonstrated that the effects of tau on MAPK signaling may be modulated by alternative splicing.

Lastly, we compared the effects of the phospho-mimicking substitutions on $0 \mathrm{~N} 3 \mathrm{R}$ and $0 \mathrm{~N} 4 \mathrm{R}$ tau. Comparing the effects of S202D on $0 N 3 R$ and $0 N 4 R$, we found that the mutation inhibited MAPK signaling to a larger extent in $0 \mathrm{~N} 4 \mathrm{R}$, where a significant decrease occurred (compare Figures 2D,E). However, when the effects of the double mutant S199D/S202D were compared between $0 \mathrm{~N} 3 \mathrm{R}$ and $0 \mathrm{~N} 4 \mathrm{R}$, we found that while $0 \mathrm{~N} 3 \mathrm{R}$
Table 1 | Phospho-mimicking mutations in 0N3R tau, tested for their ability to potentiate MAPK activation.

Ability to potentiate MAPK activation

Phospho-Ser214 (S214D)

Phospho-Ser202 (S202D)

Phospho-Ser199/Ser202 (S199D/S202D)

Phospho-Ser404 (S404D)

Phospho-Ser396/Ser404 (S396D/S404D)

Phospho-Thr231 (T231D)

(Bolded larger arrows indicate a statistically significant difference relative to non-phosphorylated tau; non-bolded smaller arrows indicate a trending result.)

S199D/S202D resembled 0N3R S202D in its ability to decrease MAPK signaling (Figure 2D), 0N4R S199D/S202D appeared to rescue MAPK signaling, yielding levels similar to wild-type $0 \mathrm{~N} 4 \mathrm{R}$ tau (Figure 2E). These findings suggest that in $0 \mathrm{~N} 4 \mathrm{R}$, while the phosphorylation of tau at Ser202 decreased the ability of tau to potentiate MAPK activation, additional phosphorylation at Ser199 neutralized the effect, returning MAPK activation levels to WT tau levels. These data indicated that the effect of phosphorylation on NGF-induced signaling depended on the tau isoform used.

Our data shows that phosphorylation differentially affected the function of $3 \mathrm{R}$ and $4 \mathrm{R}$ tau isoforms. This result resembles previous data reported for the interaction between tau and the $\mathrm{SH} 3$ domain of Fyn, where we found that phosphorylation differentially affected the equilibrium binding constant of $0 \mathrm{~N} 3 \mathrm{R}$ and $0 \mathrm{~N} 4 \mathrm{R}$ tau for the SH3 domain of Fyn (30). For the Fyn SH3 interaction, phosphorylation at Ser199/Ser202 or at Ser396/Ser404 increased 
the binding of $0 \mathrm{~N} 4 \mathrm{R}$ to the Fyn SH3 domain whereas phosphorylation at Ser199/Ser202 decreased the binding of 0N3R to the Fyn SH3 (phosphorylation at Ser396/Ser404 did not affect the binding of $0 \mathrm{~N} 3 \mathrm{R}$ to Fyn SH3). While the details of the effects of phosphorylation are not similar, both the $\mathrm{SH} 3$ binding data and the MAPK activation data demonstrate that phosphorylation differentially affected the function of $0 \mathrm{~N} 3 \mathrm{R}$ and $0 \mathrm{~N} 4 \mathrm{R}$ tau isoforms. Such findings might help explain why disease could be caused by overexpressing $4 \mathrm{R}$ tau relative to $3 \mathrm{R}$ tau. In our data, we noted that phosphorylation at Ser199/Ser202 on $4 \mathrm{R}$ tau resembled WT tau in its ability to potentiate MAPK signaling whereas the similar modification on $3 \mathrm{R}$ tau reduced MAPK activation.

During development, tau phosphorylation changes, with phosphorylation at Ser199, Ser202, Ser214, and Ser404 first increasing, then decreasing while phosphorylation at Thr231 and Ser396 remained unchanged (31). The expression of $4 \mathrm{R}$ tau was also up-regulated during development as $0 \mathrm{~N} 3 \mathrm{R}$ tau was the only tau isoform expressed in fetal brain while the remaining isoforms were expressed in an adult specific manner (32). In our experimental system where NGF was added to initiate neuronal differentiation, phosphorylation could either potentiate or attenuate MAPK activation [Figure 2, Ref. (19)]. Phosphorylation at two sites (Ser214 and Ser396/Ser404) significantly down-regulated activation while that at one site (Thr231) up-regulated activation. Therefore, the exact effects of phosphorylation would depend on the quantity of specific phosphorylated tau forms present. This, in turn, would depend on the rate of phosphorylation and dephosphorylation of tau at specific sites. However, the spatial localization of the various phosphorylated species may also be important. Since we measure MAPK activation in a transfected cell, it is possible that the spatial localization of the tau expressed by transfection may not duplicate that of the endogenous tau. If the ability of tau, expressed by transfection, to affect MAPK activation was dependent on a spatial localization not duplicated by endogenous tau, caution needs to be exercised in the interpretation of our results. Nevertheless, a critical role for tau in MAPK activation was confirmed by our experiments where ERK1/2 activation was examined without the transfection of MAPK reporter plasmids (19).

Our tests have investigated the ability of disease-related tau phosphorylation to affect the ability of tau to upregulate MAPK signaling. Among the sites we have investigated, Thr231 was the site whose phosphorylation occurred earliest during neurodegeneration $(33,34)$. Phosphorylation at Ser262/Ser356 occurred next, with Ser214 close behind; phosphorylation at Ser199/Ser202 and Ser396/Ser404 accumulated latest during neurodegeneration (33). Therefore, as tau phosphorylation changed during disease progression, tau function would similarly change. Our data suggested that phospho-Thr231-tau would potentiate MAPK activation and since phospho-Thr231 occurred early during the neurodegenerative process (20), one could speculate that MAPK activation would also occur. Data reporting the presence of activated ERK1/2 in pretangle neurons and in Braak stage I-III brains (35) supports the hypothesis that phospho-Thr231-tau may potentiate MAPK activation early in the neurodegenerative process. Then, as tau phosphorylation changed during the neurodegenerative process, the capacity for tau to potentiate MAPK activation would also change. Phosphorylation at Ser262/Ser356 would not affect MAPK activation (19) whereas phosphorylation at Ser214 and Ser396/Ser404 would lead to a decrease in the ability of tau to upregulate MAPK activation (Figure 2). In tauopathies where the level of $4 \mathrm{R}$ tau was increased, the isoform change alone might decrease MAPK activation. However, one could also speculate that the phosphorylation of $4 \mathrm{R}$ tau, for instance at Ser199/Ser202, could lead to an increase in MAPK activation, relative to that conducted by similarly phosphorylated 3R tau forms. To further investigate the relationship between tau phosphorylation and MAPK activation during neurodegeneration, tauopathy mouse models and/or human postmortem brain tissue would be probed for phospho-Thr231-tau and activated MAPK. Using immunocytochemistry, if activated MAPK only appeared in the same neurons that were positive for phospho-Thr231 tau, this would suggest that phosphorylation of tau at Thr231 was related to MAPK activation. One would also look for a correlation between activated MAPK and phosphoSer199/Ser202 in 4R tau isoforms, in addition to a correlation between phospho-Ser214-tau (or phospho-Ser396/Ser404) and a reduction in activated MAPK.

The activation of MAPK can occur in signal transduction pathways where cell proliferation is upregulated [reviewed by Ref. $(36,37)]$. Therefore, increasing the activation of MAPK could cause an increase in cell cycle activation. In Drosophila, the ability of tau to cause neurodegeneration was shown to involve cell cycle components (17). Our data supports the hypothesis that tau can potentiate cell cycle mechanisms. In a post-mitotic neuron, the activation of cell division would lead to neurodegeneration [reviewed by Ref. (38)].

It is not known whether tau participates in signal transduction in adult neurons. Gene expression in the tau-depleted mouse was compared to that of WT mouse, using microarray analysis of 8 -week-old mice (39). In the tau-depleted mouse, the genes with the highest increase in expression were FosB and c-fos [see Supplemental Data in Ref. (39)]. Since MAPK activation drives fos activation, our data leads us to speculate that the tau-depleted mouse had increased fos expression as a compensatory measure. Since the comparison had used an 8-week-old mouse, it is possible that tau was also necessary for signal transduction in the adult.

While it is clear that phosphorylated tau can form oligomers, we have not determined if the tau that upregulates MAPK activation is a monomer, dimer, trimer, or oligomer. Moreover, the mechanism by which tau affects NGF signaling is under investigation. The ability of proteins to form dimers during signal transduction processes is not unusual and in some cases, dimer formation is linked to phosphorylation. It would be important to determine if tau dimerization or oligomerization occurred in the same manner as disease-related phosphorylation, where dimerization or oligomerization would occur normally during development, then become down-regulated in the adult. If tau oligomers are found during normal development, the toxicity of tau oligomers during neurodegeneration may be related to specific 
tau oligomer functions that were inappropriate or abnormal for adult neurons.

\section{ACKNOWLEDGMENTS}

Dr. Chad Leugers supervised the work and trained Mr. Koh and Mr. Hong. Mr. Koh, and Mr. Hong performed the experiments

\section{REFERENCES}

1. Rapoport M, Dawson HN, Binder LI, Vitek MP, Ferreira A. Tau is essential to beta-amyloid-induced neurotoxicity. Proc Natl Acad Sci U $S$ A (2002) 99(9):6364-9. doi:10. 1073/pnas.092136199

2. Roberson ED, Scearce-Levie K, Palop JJ, Yan F, Cheng IH, Wu T, et al. Reducing endogenous tau ameliorates amyloid beta-induced deficits in an Alzheimer's disease mouse model. Science (2007) 316:750-4. doi:10.1126/science. 1141736

3. Lee VM, Goedert M, Trojanowski JQ. Neurodegenerative tauopathies. Annu Rev Neurosci (2001) 24:1121-59. doi:10.1146/annurev. neuro.24.1.1121

4. van Swieten J, Spillantini MG. Hereditary frontotemporal dementia caused by tau gene mutations. Brain Pathol (2007) 17(1):63-73. doi:10.1111/j.1750-3639.2007. 00052.x

5. Wolfe MS. Tau mutations in neurodegenerative diseases. $J$ Biol Chem (2009) 284(10):6021-5. doi: 10.1074/jbc.R800013200

6. SantaCruz K, Lewis J, Spires T, Paulson J, Kotilinek L, Ingelsson $\mathrm{M}$, et al. Tau suppression in a neurodegenerative mouse model improves memory function. Science (2005) 309(5733):476-81. doi: 10.1126/science. 1113694

7. Spires TL, Orne JD, SantaCruz K, Pitstick R, Carlson GA, Ashe $\mathrm{KH}$, et al. Region-specific dissociation of neuronal loss and neurofibrillary pathology in a mouse model of tauopathy. Am J Pathol (2006) 168(5):1598-607. doi:10. 2353/ajpath.2006.050840

8. Wittmann CW, Wszolek MF, Shulman JM, Salvaterra PM, Lewis J, Hutton M, et al. Tauopathy in Drosophila: neurodegeneration without neurofibrillary tangles. Science (2001) 293(5530):711-4. doi: $10.1126 /$ science. 1062382

9. Kraemer BC, Zhang B, Leverenz JB, Thomas JH, Trojanowski JQ, Schellenberg GD. Neurodegeneration and defective neurotransmission in a Caenorhabditis elegans model of tauopathy. Proc Natl Acad
Sci U S A (2003) 100(17):9980-5. doi:10.1073/pnas.1533448100

10. Kimura T, Fukuda T, Sahara $\mathrm{N}$, Yamashita S, Murayama $\mathrm{M}$, Mizoroki T, et al. Aggregation of detergent-insoluble tau is involved in neuronal loss but not in synaptic loss. J Biol Chem (2010) 285(49):38692-9. doi:10.1074/jbc. M110.136630

11. Lasagna-Reeves CA, CastilloCarranza DL, Sengupta U, Clos AL, Jackson GR, Kayed R. Tau oligomers impair memory and induce synaptic and mitochondrial dysfunction in wild-type mice. Mol Neurodegener (2011) 6:39. doi:10.1186/1750-1326-6-39

12. Tai HC, Serrano-Pozo A, Hashimoto T, Frosch MP, Spires-Jones TL, Hyman BT. The synaptic accumulation of hyperphosphorylated tau oligomers in Alzheimer disease is associated with dysfunction of the ubiquitin-proteasome system. Am J Pathol (2012) 181(4):1426-35. doi: 10.1016/j.ajpath.2012.06.033

13. Maeda S, Sahara N, Saito Y, Murayama S, Ikai A, Takashima A. Increased levels of granular tau oligomers: an early sign of brain aging and Alzheimer's disease. Neurosci Res (2006) 54(3):197-201. doi: 10.1016/j.neures.2005.11.009

14. Berger Z, Roder H, Hanna A, Carlson A, Rangachari V, Yue M, et al. Accumulation of pathological tau species and memory loss in a conditional model of tauopathy. $\mathrm{J} \mathrm{Neu}$ rosci (2007) 27(14):3650-62. doi:10. 1523/JNEUROSCI.0587-07.2007

15. Patterson KR, Remmers C, Fu Y, Brooker S, Kanaan NM, Vana L, et al. Characterization of prefibrillar tau oligomers in vitro and in Alzheimer disease. J Biol Chem (2011) 286(26):23063-76. doi:10. 1074/jbc.M111.237974

16. Lasagna-Reeves CA, CastilloCarranza DL, Sengupta U, Sarmiento J, Troncoso J, Jackson GR, et al. Identification of oligomers at early stages of tau aggregation in Alzheimer's disease. FASEB J (2012) 26(5):1946-59. doi:10.1096/fj.11-199851

17. Khurana V, Lu Y, Steinhilb ML, Oldham S, Shulman JM, Feany

shown. Dr. Lee supervised Dr. Leugers and wrote the manuscript. This work was supported by NIA RO1 AG17753, NIA R21 AG31562, and the Helen Johnson Scholars Program at The University of Iowa. The research was performed in the absence of any commercial or financial relationships that could be construed as a potential conflict of interest.

MB. TOR-mediated cell-cycle activation causes neurodegeneration in a Drosophila tauopathy model. Curr Biol (2006) 16(3):230-41. doi:10. 1016/j.cub.2005.12.042

18. Steinhilb ML, Dias-Santagata D, Fulga TA, Felch DL, Feany MB. Tau phosphorylation sites work in concert to promote neurotoxicity in vivo. Mol Biol Cell (2007) 18(12):5060-8. doi:10.1091/ mbc.E07-04-0327

19. Leugers CJ, Lee G. Tau potentiates nerve growth factor-induced mitogen-activated protein kinase signaling and neurite initiation without a requirement for microtubule binding. J Biol Chem (2010) 285(25):19125-34. doi:10.1074/jbc. M110.105387

20. Vincent I, Zheng JH, Dickson DW, Kress Y, Davies P. Mitotic phosphoepitopes precede paired helical filaments in Alzheimer's disease. Neurobiol Aging (1998) 19(4):287-96. doi:10.1016/S01974580(98)00071-2

21. Pittman RN, Wang S, DiBenedetto AJ, Mills JC. A system for characterizing cellular and molecular events in programmed neuronal cell death. J Neurosci (1993) 13(9):3669-80.

22. Otvos L Jr, Feiner L, Lang E, Szendrei GI, Goedert M, Lee VM. Monoclonal antibody PHF-1 recognizes tau protein phosphorylated at serine residues 396 and 404. J Neurosci Res (1994) 39(6):669-73. doi: 10.1002/jnr.490390607

23. Mercken M, Vandermeeren $M$, Lubke U, Six J, Boons J, Van de Voorde A, et al. Monoclonal antibodies with selective specificity for Alzheimer tau are directed against phosphatasesensitive epitopes. Acta Neuropathol (1992) 84(3):265-72. doi:10.1007/BF00227819

24. Carmel G, Mager EM, Binder LI, Kuret J. Structural basis of monoclonal antibody Alz50's selectivity for Alzheimer's disease pathology. $J$ Biol Chem (1996) 271(51):32789-95. doi:10.1074/jbc.271.51.32789

25. Horowitz PM, Patterson KR, Guillozet-Bongaarts AL, Reynolds MR, Carroll CA, Weintraub ST, et al. Early N-terminal changes and caspase- 6 cleavage of tau in Alzheimer's disease. J Neurosci (2004) 24(36):7895-902. doi:10. 1523/JNEUROSCI.1988-04.2004

26. Watanabe A, Hasegawa M, Suzuki M, Takio K, Morishima-Kawashima $\mathrm{M}$, Titani $\mathrm{K}$, et al. In vivo phosphorylation sites in fetal and adult rat tau. J Biol Chem (1993) 268(34):25712-7.

27. Morishima-Kawashima $M$, Hasegawa M, Takio K, Suzuki M, Yoshida H, Titani K, et al. Prolinedirected and non-proline-directed phosphorylation of PHF-tau. J Biol Chem (1995) 270:823-9. doi:10.1074/jbc.270.2.823

28. Niblock M, Gallo JM. Tau alternative splicing in familial and sporadic tauopathies. Biochem Soc Trans (2012) 40(4):677-80. doi:10. 1042/BST20120091

29. D’Souza I, Schellenberg GD. Regulation of tau isoform expression and dementia. Biochim Biophys Acta (2005) 1739:104-15. doi:10.1016/j. bbadis.2004.08.009

30. Bhaskar K, Yen SH, Lee G. Disease related modifications in tau affect the interaction between Fyn and tau. $J$ Biol Chem (2005) 280:35119-25. doi:10.1074/jbc.M505895200

31. Yu Y, Run X, Liang Z, Li Y, Liu F, Liu Y, et al. Developmental regulation of tau phosphorylation, tau kinases, and tau phosphatases. $J$ Neurochem (2009) 108(6):1480-94. doi:10.1111/j.1471-4159.2009. 05882.x

32. Goedert M, Spillantini MG, Potier MC, Ulrich J, Crowther RA. Cloning and sequencing of the cDNA encoding an isoform of microtubule associated protein tau containing four tandem repeats: differential expression of tau protein mRNAs in human brain. EMBO $J$ (1989) 8(2): 393-9.

33. Augustinack JC, Schneider A, Mandelkow EM, Hyman BT. Specific tau phosphorylation sites correlate with severity of neuronal cytopathology in Alzheimer's disease. Acta Neuropathol (2002) 103(1):26-35. doi: $10.1007 / \mathrm{s} 004010100423$ 
34. Luna-Munoz J, Chavez-Macias L, Garcia-Sierra F, Mena R. Earliest stages of tau conformational changes are related to the appearance of a sequence of specific phospho-dependent tau epitopes in Alzheimer's disease. J Alzheimers Dis (2007) 12(4): 365-75.

35. Pei JJ, Braak H, An WL, Winblad B, Cowburn RF, Iqbal K, et al. Up-regulation of mitogenactivated protein kinases ERK1/2 and MEK $1 / 2$ is associated with the progression of neurofibrillary degeneration in Alzheimer's disease. Brain Res Mol Brain Res (2002) 109(1-2):45-55. doi:10.1016/ S0169-328X(02)00488-6
36. Rubinfeld H, Seger R. The ERK cascade: a prototype of MAPK signaling. Mol Biotechnol (2005) 31(2):151-74. doi:10.1385/MB:31: 2:151

37. Chang L, Karin M. Mammalian MAP kinase signalling cascades. Nature (2001) 410(6824):37-40. doi: $10.1038 / 35068583$

38. Yang Y, Herrup K. Cell division in the CNS: protective response or lethal event in post-mitotic neurons? Biochim Biophys Acta (2007) 1772(4): 457-66. doi:10.1016/j.bbadis.2006. 10.002

39. Oyama F, Kotliarova S, Harada A, Ito M, Miyazaki $H$, Ueyama Y, et al. Gem GTPase and tau: morphological changes induced by gem GTPase in cho cells are antagonized by tau. $J$ Bio Chem (2004) 279(26):27272-7. doi:10.1074/jbc.M401634200

Conflict of Interest Statement: The authors declare that the research was conducted in the absence of any commercial or financial relationships that could be construed as a potential conflict of interest.

Received: 25 July 2013; paper pending published: 08 August 2013; accepted: 01 October 2013; published online: 17 October 2013.
Citation: Leugers CJ, Koh JY, Hong $W$ and Lee G (2013) Tau in MAPK activation. Front. Neurol. 4:161. doi: 10.3389/fneur.2013.00161

This article was submitted to Neurodegeneration, a section of the journal Frontiers in Neurology.

Copyright (c) 2013 Leugers, Koh, Hong and Lee. This is an open-access article distributed under the terms of the Creative Commons Attribution License (CC $B Y)$. The use, distribution or reproduction in other forums is permitted, provided the original author(s) or licensor are credited and that the original publication in this journal is cited, in accordance with accepted academic practice. No use, distribution or reproduction is permitted which does not comply with these terms. 